\title{
DIMENSIONES LATENTES DE LA DIFERENCIACIÓN DEL ESPACIO SOCIAL EN UNA METRÓPOLIS LATINOAMERICANA. EL CASO DEL GRAN SANTIAGO ${ }^{1}$
}

\author{
Jorge ORTIZ VELIZ y Paulina SCHIAPPACASSE CAMBIASO \\ Departamento de Geografía - Facultad de Arquitectura y Urbanismo \\ Universidad de Chile
}

\begin{abstract}
Resumen: A la luz de un conjunto de variables socioeconómicas, demográficas, de movilidad espacial de la población y características de la vivienda, se analizan las dimensiones latentes del espacio social de la capital nacional y se relacionan las estructuras resultantes con los modelos sociales de la diferenciación espacial propuestos para ciudades de carácter metropolitano de latinoamérica.
\end{abstract}

Palabras clave: dimensiones latentes, espacio social.

\begin{abstract}
In the light of a set of socioeconomical, demographia, population spatial mobility and building characteristics variables, the latent dimensions of the social space of the national capital are analysed and related with the structures linked to the social models of spatial diferentation proposed for cities of metropolitan character in Latinoamerica.
\end{abstract}

Key words: latent dimensions, social space.

\section{INTRODUCCIÓN}

Dentro de la problemática espacial intraurbana, uno de los aspectos que $h$ a concitado una gran atención por parte de investigadores de diversas disciplinas, dice relación con la estructuración diferencial del espacio social al interior de las ciudades.

Dichas diferenciaciones se expresan básicamente en términos de dimensiones latentes, es decir, de estructuras con características muy bien definidas, tanto en atributos como expresión espacial, las que son productos de acciones socioeconómicas, demográficas y culturales. Al respecto, cuando estas diferenciaciones se repiten en el tiempo, o bien cuando se expresan espacialmente de manera similar, tal como ocurre en

${ }^{1}$ Proyecto Fondecyt n 1970474. 
las urbes de países desarrollados, se puede llegar a establecer ciertas regularidades en el desarrollo socioespacial de las ciudades, regularidades que al final de cuenta constituyen la base de elaboración de modelos urbanos.

En este orden de ideas y teniendo en consideración que estudios en esta línea de trabajos son escasos en Latinoamérica, y en particular en Chile, se desea establecer las dimensiones fundamentales del espacio social de una ciudad de carácter metropolitano, cuya primacía urbana lejos de atenuarse en el tiempo ha tendido más bien a acentuarse.

\section{MARCO TEÓRICO Y OBJETIVOS}

Existe una literatura bastante amplia sobre estudios de diferenciación social intraurbana, particularmente en países desarrollados. En este sentido, mención especial merece los Estados Unidos, con la presencia de centros universitarios de reconocido prestigio como la llamada Escuela Ecológica de Chicago de los años 20, o bien la Escuela de Los Angeles con sus más conspicuos representantes, E.Shevky, M.Williams y W.Bell.

Los trabajos generados hasta la década de los 40 estuvieron esencialmente caracterizados por una extrapolación y proyección analógica de la ecología biológica, tanto es así que Park en 1936 (THEODORSON, 1974) señalaba que el proceso básico de las relaciones humanas era la competencia, la cual implicaba la lucha por el espacio. La competencia, aparece como el principio organizativo fundamental en las comunidades humanas, pero a diferencia de las comunidades vegetales y animales, ésta conlleva un grado automático y no planificado de cooperación. En otras palabras como lo señala TIMMS (1976), es el proceso de competencia impersonal el que proporciona el marco de la estructura ecológica.

El mismo esquema analógico, aunque a menor escala, presenta el trabajo de McKenzie reproducido por Theodorson, en que aparte de aceptar el concepto de competencia como proceso ecológico, desarrolla una amplia conceptualización biológica con respecto al dominio por determinados espacios al interior de la ciudad.

En 1950, Myers (THEODORSON, 1974) critica los planteamientos de Park, a 1 señalar que la competencia no es impersonal, sino que por el contrario, es absolutamente personal y deliberada. Los hombres compiten no de un modo abstracto, sino como seres humanos dentro de un marco sociocultural en donde los usos y los valores son instrumentos que regulan el proceso competitivo. Esta crítica, al igual que la de 
Gettys (THEODORSON, 1974), está orientada a la excesiva analogía de la ecología natural frente a los problemas de orden social.

Esta misma década conoció un período de expansión en estudios de ecología urbana lo que ha permitido en la actualidad algunas conclusiones de carácter general, a pesar de las diferencias concretas que se pueden observar en la estructura del espacio social de las distintas ciudades en que se han llevado adelante investigaciones de esta índole. En efecto, los estudios han llegado a identificar, lo que BERRY (1975) y sus discípulos han conceptualizado como las dimensiones latentes del espacio social intraurbano (socioeconómica, familiar y étnica), materizlizándose cada una de ellas en una expresión espacial claramente definida.

Estas dimensiones en poco difieren con las establecidas por Shevky y Bell, tempranamente para la ciudad de Los Angeles. Para dichos investigadores, las agrupaciones urbanas no son concebidas como unidades aisladas, autosuficientes, sino como partes de un sistema de relaciones más extensas. Partiendo de la tipología de las áreas sociales, llegaron a un esquema de clasificación en el que las poblaciones quedan categorizadas en términos de tres factores o rangos básicos (rango social, urbanización y segregación), los cuales determinan la diferenciación y estratificación social de la ciudad contemporánea.

Lo reseñado hasta el momento corresponde esencialmente a estudios efectuados en países desarrollados (Estados Unidos, Canadá, Suecia, Nueva Zelanda, entre otros), por lo tanto las generalizaciones que algunos autores han expresado con respecto al comportamiento del espacio social urbano, se reducen exclusivamente a esas realidades, lo cual conlleva a suponer que los factores identificados no proporcionan un marco de referencia definitivo en el caso de los países en vía de desarrollo.

Al decir de ORTIZ (1980) y BOADA (1985) lo anteriormente expresado se ve avalado por la escasez de trabajos en esta línea en América Latina, reconociéndose particularmente en este sentido el estudio efectuado en Río de Janeiro por Morris y Pyle (BERRY, 1975).

En Chile, destaca lo realizado por BÄHR y RIESCO (1981) para la ciudad de Santiago, al identificar dos factores (status socioeconómico y status familiar), dimensiones que inciden fuertemente en la ecológica social de la capital. Más recientemente, SCHIAPPACASSE (1998) logra caracterizar el espacio social del Gran Santiago a partir de 5 factores con connotaciones socioeconómicas, familiares y de vivienda.

Por otro lado, y en el marco de modelos de crecimiento urbano propuestos para ciudades latinoamericanas, con una fuerte componente socioecológica, mención especial 
merecen los planteamientos de GRIFFIN y FORD (1980), BÄHR y MERTINS (1982 y 1993) y FORD (1996), quienes han desarrollado "esquemas ideales" respecto de la ciudad latinoamericana, y que ha servido de guía a sus autores para una exposición global de la estructura funcional y socioespacial de éstas.

En virtud de lo anteriormente expuesto, el presente estudio tiene como objetivo general, identificar las dimensiones latentes que estructuran el espacio social del Gran Santiago y relacionar los patrones resultantes con los modelos socioespaciales urbanos propuestos para las metrópolis latinoamericanas.

\section{METODOLOGÍA}

A fin de alcanzar los objetivos propuestos en la presente investigación se llevaron adelante los siguientes pasos metodológicos:

- Extracción de variables demográficas, económicas y de vivienda del Censo de Población y Vivienda del año 1992.

- Asociación de las variables a las unidades espaciales de análisis (distritos)

- Utilización del software Redatam + desarrollado por CELADE en 1988 con el fin de recuperar y procesar la información censal a nivel de los 277 distritos que conforman las 34 comunas del Gran Santiago.

- Selección de variables mediante estadística descriptiva (valores de desviación standard) y coeficientes de alineación.

- Aplicación de técnica multivariada a través del software estadístico SPSS para Windows, con el objeto de reducir número inicial de variables a unos pocos factores.

- Espacialización de las dimensiones latentes identificadas mediante el software Arc-View.

- Clasificación, espacialización y caracterización social de los distritos a partir de la técnica de análisis de agrupamientos (cluster analysis). 


\section{ESTRUCTURA INTERNA DEL ESPACIO SOCIAL DEL GRAN SANTIAGO}

- Selección de las variables: En virtud de los marcos referenciales en este tipo de estudios, se procedió a extraer del censo de población variables relativas a condiciones demográficas, económicas, educativas y de movilidad espacial de la población, además de antecedentes relativos a la vivienda.

En una primera selección, 152 fueron las variables consideradas, a las que se le calcularon indicadores de estadística descriptiva. De éstas, 94 presentaron valores 3.0 y más desviaciones standard, procediéndose a calcular los coeficientes de alineación (c.a.= 1-r2). Finalmente, se seleccionaron para el estudio 64 variables fuertemente correlacionadas y no redundantes entre sí (Tabla 1).

Tabla 1.-Variables Seleccionadas.

\begin{tabular}{|c|c|}
\hline $\mathrm{N}^{\circ}$ & NOMBRE DE LA VARIABLE \\
\hline 1 & Densidad de Población año $1982\left(\mathrm{hab} / \mathrm{km}^{2}\right)$ \\
\hline 2 & Densidad de Población año $1992\left(\mathrm{hab} / \mathrm{km}^{2}\right.$ ) \\
\hline 4 & $\%$ de hogares con teléfono \\
\hline 5 & $\%$ de viviendas material predominante techo es zinc o pizarreño \\
\hline 6 & $\%$ de viviendas material predominante piso es plástico, baldosa cemento o ladrillo \\
\hline $\begin{array}{l}7 \\
8\end{array}$ & $\begin{array}{l}\% \text { de viviendas material predominante techo es loza de hormigón, teja o tejuela de madera } \\
\% \text { de viviendas tipo departamento }\end{array}$ \\
\hline 9 & $\begin{array}{l}\% \text { de viviendas material predominante piso es parquet o entablado, baldocín cerámico o } \\
\text { alfombra muro a muro }\end{array}$ \\
\hline 10 & $\%$ de viviendas tipo casa \\
\hline 11 & $\%$ de hogares con auto particular \\
\hline 12 & $\%$ de hogares con lavadora programable \\
\hline 13 & \% de viviendas material predominante pared es madera o tabique forrado \\
\hline 14 & $\%$ de viviendas material predominante pared es ladrillo o concreto \\
\hline 15 & $\%$ de hogares con TV color \\
\hline 16 & $\%$ de hogares con videograbador \\
\hline 17 & $\%$ de viviendas disponen de alcantarilla o fosa \\
\hline 22 & $\%$ de viviendas disponen de pozo negro o cajón en acequia \\
\hline 23 & \% de población con estudios básicos \\
\hline 24 & $\%$ de viviendas material predominante pared es adobe o barro empajado \\
\hline 25 & $\%$ de hogares con microondas \\
\hline 27 & $\%$ de viviendas arrendadas \\
\hline 31 & $\%$ de viviendas abastecidas por pozo o noria \\
\hline 33 & $\%$ de población con estudios universitarios \\
\hline 34 & $\%$ Oficiales, Operarios y Artesanos de Artes Mecánicas y de Otros Oficios \\
\hline 35 & $\%$ de mujeres entre $21-30$ años con 1 o 2 hijos nacidos vivos \\
\hline 36 & $\%$ de viviendas tipo mejora o mediagua, rancho, choza o ruca \\
\hline 37 & $\%$ de población con estudios medios \\
\hline 38 & $\%$ de mujeres que estaban trabajando con ingreso semana anterior censo \\
\hline 39 & $\%$ de mujeres entre $14-20$ años con 1 o 2 hijos nacidos vivos \\
\hline 40 & $\%$ Profesionales \\
\hline 41 & $\%$ de mujeres que trabajaban en quehaceres del hogar semana anterior censo \\
\hline 43 & \% de población que trabaja en la Industria Manufacturera \\
\hline 44 & $\%$ de viviendas cedidas o gratuítas \\
\hline
\end{tabular}




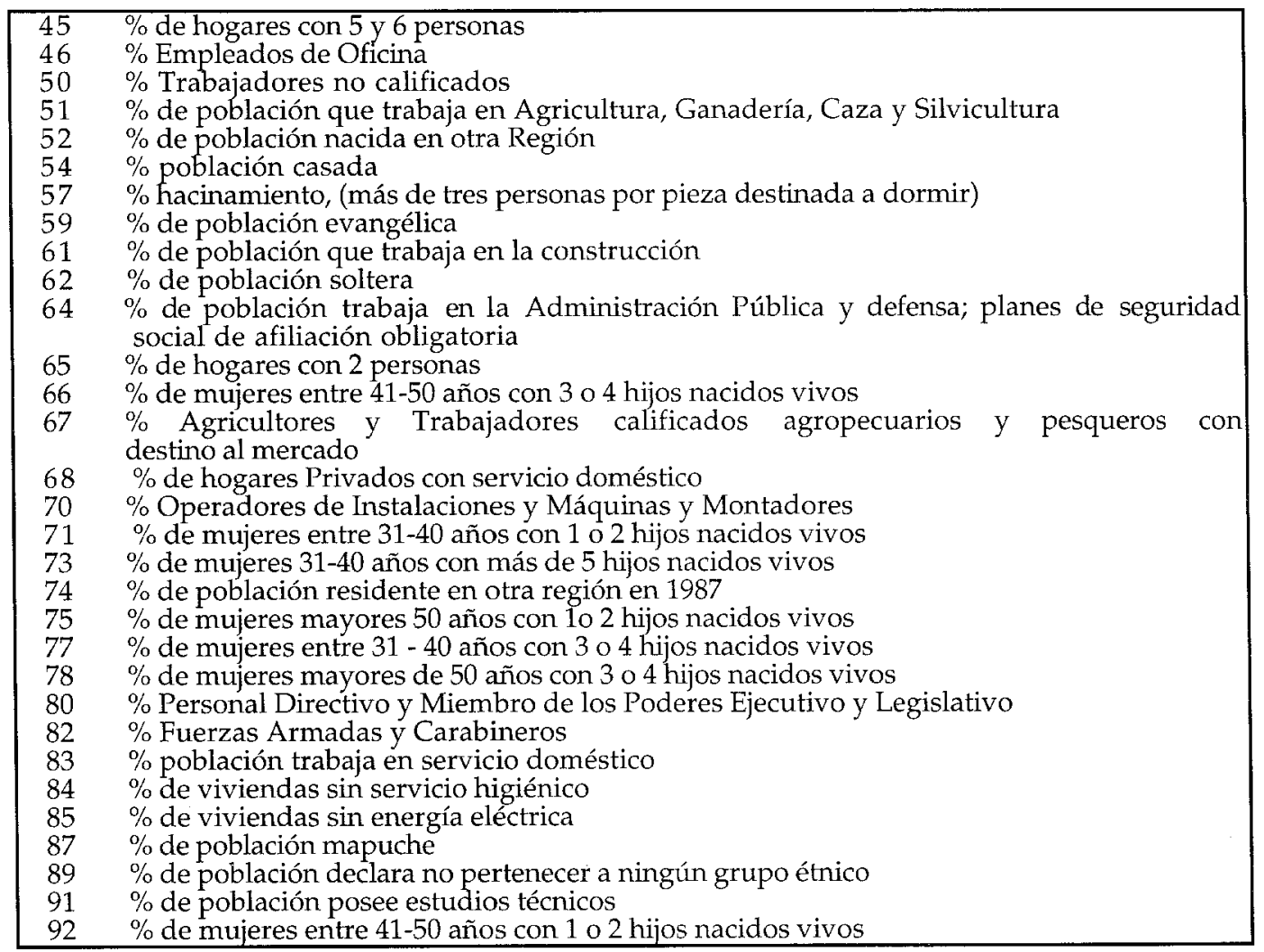

Nota: Se han mantenido las numeraciones iniciales de las variables

- Análisis de los resultados: La aplicación del análisis factorial a la matriz geográfica conformada por 277 unidades. de observación y 64 variables originales, permitió reducir a éstas a 11 variables complejas o factores que explican más del 93\% de la varianza total (Tabla 2).

Teniendo en cuenta que el objetivo del análisis factorial es maximizar la varianza explicada con el mínimo número de factores, se procedió al criterio expresado por Bravo (1979) de trabajar con el número de factores que en conjunto alcancen el $70 \%$ de la explicación de la varianza común de las variables, es decir, con los 5 primeros los cuales suman el $72.8 \%$. 
Tabla 2.- Valores Propios y Varianza Explicada.

\begin{tabular}{|cccc|}
\hline Factor & Eigenvalue & $\begin{array}{c}\text { Porcentaje Simple } \\
\text { de la Varianza }\end{array}$ & $\begin{array}{c}\text { Porcentaje Acumulado } \\
\text { de la Varianza }\end{array}$ \\
\hline 1 & 22.4 & 35.0 & 35.0 \\
2 & 7.5 & 11.7 & 46.7 \\
3 & 6.0 & 9.3 & 56.0 \\
4 & 6.0 & 9.3 & 65.3 \\
5 & 4.8 & 7.5 & 72.8 \\
6 & 3.7 & 5.7 & 78.5 \\
7 & 2.8 & 4.3 & 82.8 \\
8 & 2.5 & 3.9 & 86.7 \\
9 & 1.7 & 2.6 & 89.3 \\
10 & 1.5 & 2.3 & 91.6 \\
11 & 1.2 & 1.8 & 93.4 \\
\hline
\end{tabular}

- Interpretación de los factores: La interpretación de los factores se efectuó a partir de la matriz de carga factorial (Tabla 3) teniendo en consideración los pesos más altos de los factores en las variables, independiente del sentido del signo de estas. Resultado de ello los factores se denominaron de la siguiente forma:

Factor 1 (Socioeconómico): Este primer factor denominado Socioeconómico está caracterizado positivamente con hogares que cuentan con auto particular (0.968), lavadora programable (0.967), microondas (0.947), videograbador (0.942), y de poseer la población estudios universitarios (0.936) y ser profesionales $(0.915)$.

Por el contrario, valores negativos altos en este factor se dan en variables relativas a mujeres que trabajan en quehaceres del hogar (-0.854), operarios y artesanos que cumplen labores de artes mecánicas (-0.848), y proporción de población mapuche (-0.815).

Factor 2 (Suburbanización): Caracteriza positivamente a las entidades espaciales cuya población cumple labores primarias (0.932), particularmente agrícolas (0.900), y cuyas viviendas son abastecidas por pozo o noria (0.881), que disponen de pozo negro como sistema de eliminación de excretas (0.878) y carecen de energía eléctrica $(0.830)$. Recíprocamente el valor más alto negativo viene dado por las viviendas que cuentan con alcantarillado $(-0.764)$.

Factor 3 (Nivel ocupacional): Tipifica a los distritos en donde predominan los empleados de oficina (0.806), la población con niveles de estudios medios (0.653) y 
técnicos $(0.635)$ y negativamente la presencia de trabajadores no calificados $(-0.876)$ y que trabajan en construcción (0.587).

Factor 4 (Familiar): Este factor queda caracterizado positivamente por hogares compuestos por 5 y 6 personas (0.842) y viviendas tipo casa (0.834). Los valores negativos vienen representados por viviendas multifamiliares o apartamentos ($0.777)$, hogares con 2 personas $(-0.716)$ y población nacida en otra región $(-0.647)$.

Tabla 3.- Matriz de Carga Factorial.

\begin{tabular}{|c|c|c|c|c|c|}
\hline $\mathrm{N}^{\circ}$ de la variable & Factor 1 & Factor 2 & Factor 3 & Factor 4 & Factor 5 \\
\hline V1 & $-0,232$ & $-0,245$ & 0,022 & 0,118 & 0,063 \\
\hline $\mathrm{V} 2$ & $-0,275$ & $-0,312$ & 0,006 & 0,047 & $-0,075$ \\
\hline V4 & 0,871 & $-0,218$ & 0,211 & 0,164 & 0,236 \\
\hline V5 & $-0,845$ & 0,060 & 0,012 & $-0,372$ & 0,068 \\
\hline V6 & $-0,524$ & $-0,128$ & $-0,019$ & $-0,200$ & $-0,690$ \\
\hline V7 & 0,887 & $-0,106$ & 0,017 & 0,299 & $-0,051$ \\
\hline V8 & 0,418 & $-0,141$ & 0,073 & $-0,777$ & $-0,071$ \\
\hline V9 & 0,525 & 0,064 & 0,052 & 0,104 & 0,727 \\
\hline V10 & $-0,208$ & $-0,050$ & 0,072 & 0,834 & $-0,068$ \\
\hline V11 & 0,968 & $-0,106$ & 0,010 & $-0,038$ & $-0,052$ \\
\hline V12 & 0,967 & $-0,111$ & $-0,015$ & $-0,037$ & 0,054 \\
\hline V13 & $-0,495$ & 0,499 & $-0,394$ & $-0,199$ & $-0,193$ \\
\hline V14 & 0,528 & $-0,477$ & 0,318 & 0,043 & $-0,396$ \\
\hline V15 & 0,731 & $-0,277$ & 0,407 & $-0,034$ & 0,077 \\
\hline V16 & 0,942 & $-0,184$ & 0,108 & $-0,053$ & 0,055 \\
\hline V17 & 0,293 & $-0,764$ & 0,202 & 0,054 & 0,001 \\
\hline V22 & $-0,247$ & 0,878 & $-0,219$ & $-0,106$ & $-0,018$ \\
\hline V23 & $-0,731$ & 0,332 & $-0,475$ & $-0,203$ & $-0,191$ \\
\hline V24 & $-0,114$ & $-0,062$ & 0,117 & 0,045 & 0,907 \\
\hline V25 & 0,947 & $-0,095$ & $-0,198$ & $-0,042$ & 0,079 \\
\hline V27 & 0,262 & $-0,235$ & 0,216 & 0,332 & 0,735 \\
\hline V31 & 0,001 & 0,881 & $-0,001$ & $-0,004$ & $-0,026$ \\
\hline V33 & 0,936 & $-0,127$ & 0,050 & 0,212 & 0,038 \\
\hline V34 & $-0,848$ & $-0,089$ & $-0,286$ & $-0,158$ & $-0,202$ \\
\hline V35 & 0,894 & $-0,148$ & 0,056 & 0,259 & 0,111 \\
\hline V36 & $-0,478$ & 0,545 & $-0,388$ & $-0,109$ & $-0,237$ \\
\hline V37 & $-0,114$ & $-0,327$ & 0,653 & 0,008 & 0,368 \\
\hline V38 & 0,868 & $-0,260$ & $-0,015$ & 0,289 & 0,215 \\
\hline V39 & 0,687 & $-0,117$ & 0,041 & 0,136 & $-0,112$ \\
\hline V40 & 0,915 & $-0,119$ & 0,107 & 0,240 & 0,056 \\
\hline V41 & $-0,854$ & 0,259 & $-0,017$ & $-0,245$ & $-0,198$ \\
\hline
\end{tabular}




\begin{tabular}{|c|c|c|c|c|c|}
\hline V43 & $-0,778$ & $-0,116$ & 0,028 & $-0,218$ & $-0,135$ \\
\hline V44 & $-0,240$ & 0,703 & $-0,098$ & $-0,028$ & 0,078 \\
\hline V45 & 0,058 & 0,016 & $-0,125$ & 0,842 & $-0,051$ \\
\hline V46 & 0,290 & $-0,265$ & 0,806 & 0,106 & 0,141 \\
\hline V50 & 0,047 & 0,194 & $-0,876$ & $-0,187$ & $-0,102$ \\
\hline V51 & $-0,003$ & 0,932 & $-0,098$ & $-0,050$ & $-0,062$ \\
\hline V52 & 0,435 & $-0,107$ & 0,001 & $-0,647$ & 0,189 \\
\hline V54 & $-0,198$ & $-0,022$ & 0,346 & 0,446 & $-0,389$ \\
\hline V57 & $-0,697$ & 0,261 & $-0,398$ & $-0,225$ & 0,113 \\
\hline V59 & $-0,721$ & 0,143 & $-0,446$ & $-0,193$ & $-0,312$ \\
\hline V61 & $-0,593$ & $-0,039$ & $-0,587$ & $-0,074$ & $-0,369$ \\
\hline V62 & 0,498 & $-0,012$ & $-0,262$ & 0,256 & 0,155 \\
\hline V64 & 0,134 & 0,021 & 0,409 & 0,058 & 0,059 \\
\hline V65 & 0,241 & $-0,151$ & 0,232 & $-0,716$ & 0,388 \\
\hline V66 & 0,567 & $-0,317$ & 0,480 & 0,240 & 0,221 \\
\hline V 67 & $-0,063$ & 0,900 & $-0,097$ & $-0,080$ & $-0,065$ \\
\hline V68 & 0,750 & $-0,121$ & $-0,555$ & $-0,064$ & 0,040 \\
\hline V70 & $-0,742$ & $-0,007$ & 0,093 & $-0,257$ & $-0,209$ \\
\hline V71 & 0,714 & $-0,136$ & 0,130 & $-0,501$ & 0,269 \\
\hline V73 & $-0,166$ & 0,106 & $-0,429$ & 0,562 & $-0,467$ \\
\hline V74 & 0,306 & 0,030 & $-0,015$ & 0,454 & 0,228 \\
\hline V75 & 0,384 & $-0,147$ & 0,214 & $-0,540$ & 0,579 \\
\hline V77 & 0,362 & $-0,253$ & 0,500 & $-0,046$ & 0,049 \\
\hline V78 & 0,458 & $-0,401$ & 0,294 & 0,287 & 0,234 \\
\hline V80 & 0,848 & $-0,072$ & $-0,049$ & 0,118 & 0,306 \\
\hline V82 & $-0,001$ & 0,195 & 0,096 & 0,040 & $-0,021$ \\
\hline V83 & 0,826 & $-0,072$ & $-0,469$ & $-0,068$ & 0,010 \\
\hline V84 & $-0,425$ & 0,023 & $-0,179$ & $-0,086$ & 0,049 \\
\hline V85 & $-0,049$ & 0,830 & $-0,084$ & 0,027 & $-0,015$ \\
\hline V87 & $-0,815$ & $-0,141$ & $-0,161$ & $-0,120$ & $-0,179$ \\
\hline V89 & 0,835 & 0,160 & 0,105 & 0,145 & 0,036 \\
\hline V91 & $-0,343$ & $-0,314$ & 0,635 & $-0,091$ & $-0,213$ \\
\hline V92 & 0,517 & $-0,114$ & 0,230 & $-0,590$ & 0,387 \\
\hline
\end{tabular}

Nota: los pesos en negrita indican los valores más altos de la variable en el factor La denominación de las variables aparece en la Tabla 1.

Factor 5 (Vivienda): Este último factor nominado Vivienda, da cuenta en sus valores positivos del material de adobe de las paredes (0.907) y piso de parquet (0.727) y de la proporción de viviendas arrendadas (0.735). Negativamente destacan las viviendas con piso de plástico, baldosa, cemento o ladrillo $(-0.690)$. 


\section{PATRÓN SOCIOESPACIAL DE LAS DIMENSIONES LATENTES DEL GRAN SANTIAGO}

A partir de la matriz de puntaje o score, se procedió a espacializar los pesos de los factores en los respectivos distritos de la capital nacional, previo rangueo y calificación de los mismos, de tal manera que los patrones espaciales resultantes son los que se indican a continuación:

1.- Patrón Socioeconómico: La estructura resultante (Fig.1), da cuenta de una expresión espacial conforme al planteamiento de GRIFFIN y FORD (1980) en el sentido que aparece representada claramente la espina dorsal que nace en el centro de la ciudad y se proyecta hacia los contrafuertes cordilleranos a través de un corredor de distritos de status socioeconómico medios-altos, altos y muy altos de las comunas de Providencia, Nuñoa, La Reina, Las Condes, Vitacura y Lo Barnechea. La excepción a este comportamiento viene dado por el distrito Cerro Apoquindo en la comuna de Las Condes, donde se alcanza un valor medio-bajo, así como también en el distrito Aeródromo de Tobalaba con la presencia de la Villa La Reina, en la comuna del mismo nombre.

En este eje sectorial de alto status también se presenta como una excepción un peso socioeconómico muy bajo, en la medida que existe una alta proporción de viviendas que no cuentan con autos particulares, que carecen de lavadoras, microondas y videograbador y en donde la población profesional con estudios universitarios es reducida. La razón que lo explica tiene que ver con la presencia de poblamiento rural disperso en el distrito Farellones, de la comuna de Lo Barnechea, el que no obstante a su bajo peso demográfico, relaja la estructura socioeconómica del sector, tal como ocurre además con los emplazamientos marginales en las cercanías del río Mapocho.

Por otro lado, los valores medios-bajos, bajos y muy bajos, se expresan espacialmente conforme a una curva de decaimiento desde el centro a la periferia, tanto norte, sur, como poniente de la ciudad.

El anillo más exterior queda tipificado por distritos con pesos socioeconómicos muy bajos, es decir, con una alta presencia de población femenina que trabaja en quehaceres del hogar y de varones que cumplen funciones de operarios y artesanos en actividades mecánicas y en donde predomina además una alta proporción de población cuyo origen étnico es mapuche. 


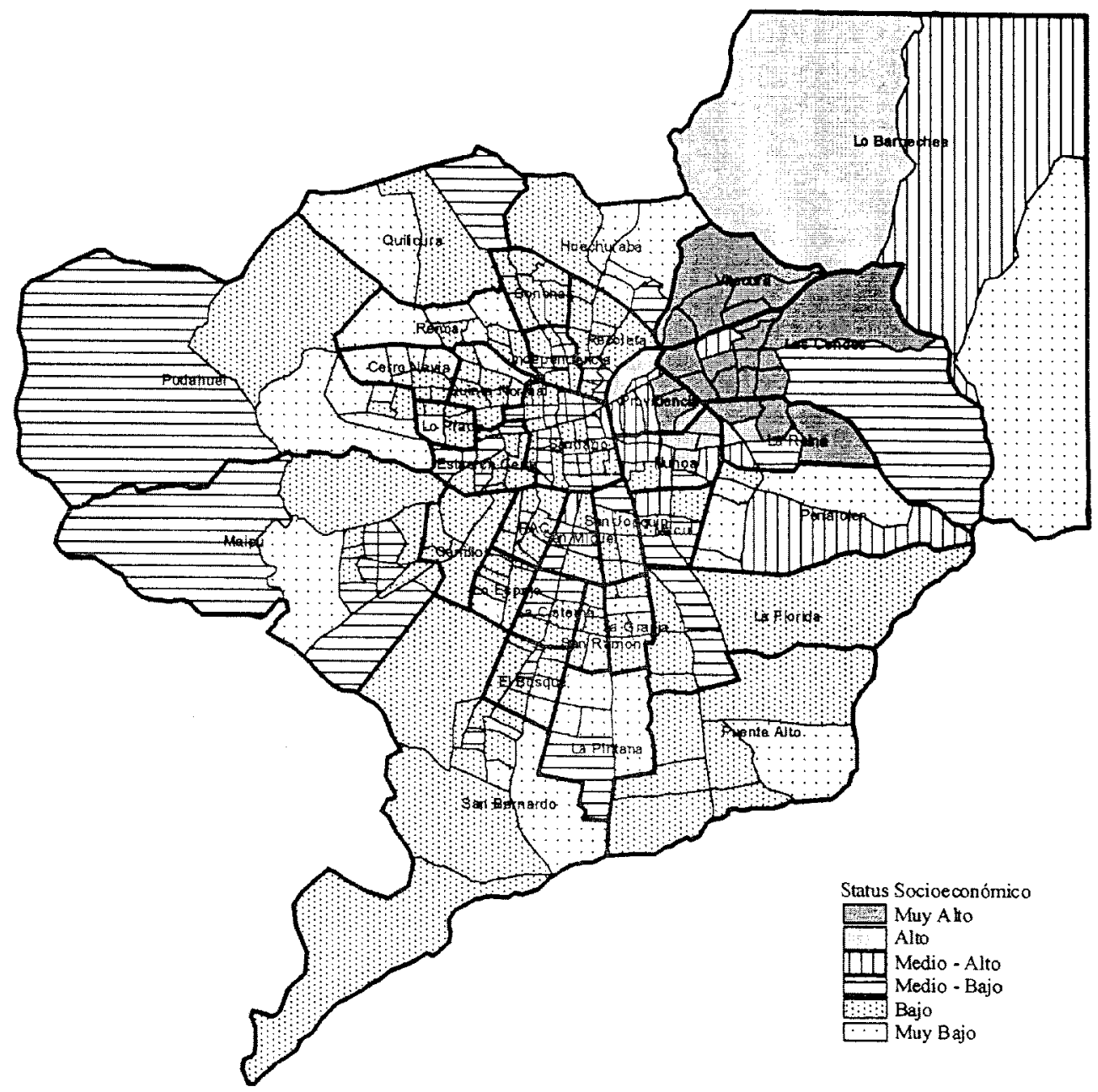

ESCALA APROX. 1: 300.000

1

Figura 1.- Gran Santiago. Patrón Socioeconómico. 1992.

(Fuente: Inostroza, 1998) 
2.- Patrón Suburbanización: La expresión espacial de esta dimensión latente del espacio social del Gran Santiago resulta ser francamente periférica (Fig.2). En efecto, la proporción más alta de distritos que presentan viviendas con altos niveles de subequipamiento de servicios básicos, quedan reflejados en el anillo más exterior de 1 a ciudad. Mención especial merecen distritos excéntricos de comunas del noroeste y suroeste, particularmente de Quilicura, Pudahuel, Maipú y San Bernardo, y que desde el punto de vista socioeconómico corresponden a entidades caracterizadas por niveles medios-bajos, bajos o bien muy bajos. Estos espacios, a su vez, vienen a corresponder a sectores de la ciudad donde el valor del suelo urbano alcanzan montos bajos de menos de $1 \mathrm{UF} / \mathrm{m}^{2}$ (ORTIZ y SCHIAPPACASSE, 1997).

Salvo algunas escasas excepciones, los territorios más céntricos y el sector oriente de la capital, quedan tipificados por presentar en general un buen nivel de servicios básicos.

3.- Patrón Nivel Ocupacional: La Figura 3 muestra la estructura del nivel ocupacional en cuanto al predominio de empleados de oficina y población con estudios medios y técnicos. Al respecto, se pueden reconocer desde el centro a la periferia de la ciudad 3 anillos siendo el más central una mezcla de distritos de valores medios, medios-bajos y bajos. En cambio el anillo inmediatamente colindante, en especial del sector poniente y sur, viene a corresponder al dominio de los montos más altos de este nivel ocupacional, para luego decaer nuevamente a pesos medios y medios-bajos.

Donde estos últimos valores se expresan más homogéneamente en el espacio, es en el sector de más alto status social, es decir, en la espina dorsal correspondiente a los distritos del oriente de la capital, expresándose espacialmente como una cuña invertida desde el centro de Santiago hacia la cordillera.

En general, este patrón no difiere sustancialmente por lo demostrado por Inostroza (1998), al plotear espacialmente la distribución de los empleados de oficina en su estudio referido al comportamiento areal de diversas categorías del status ocupacional de la población del Gran Santiago.

4.- Patrón Familiar: La expresión espacial de los atributos es conforme con el factor "estructura de hogares y de edad de la población residente" de BAHR y RIESCO (1981), en su estudio del Gran Santiago con información censal de 1970. Fn efecto, los espacios más centrales de la ciudad quedan caracterizados por el dominio de la población que habita en viviendas tipo apartamentos, con hogares pequeños (2 personas) y población nacida en otra región del país (Fig. 4). 


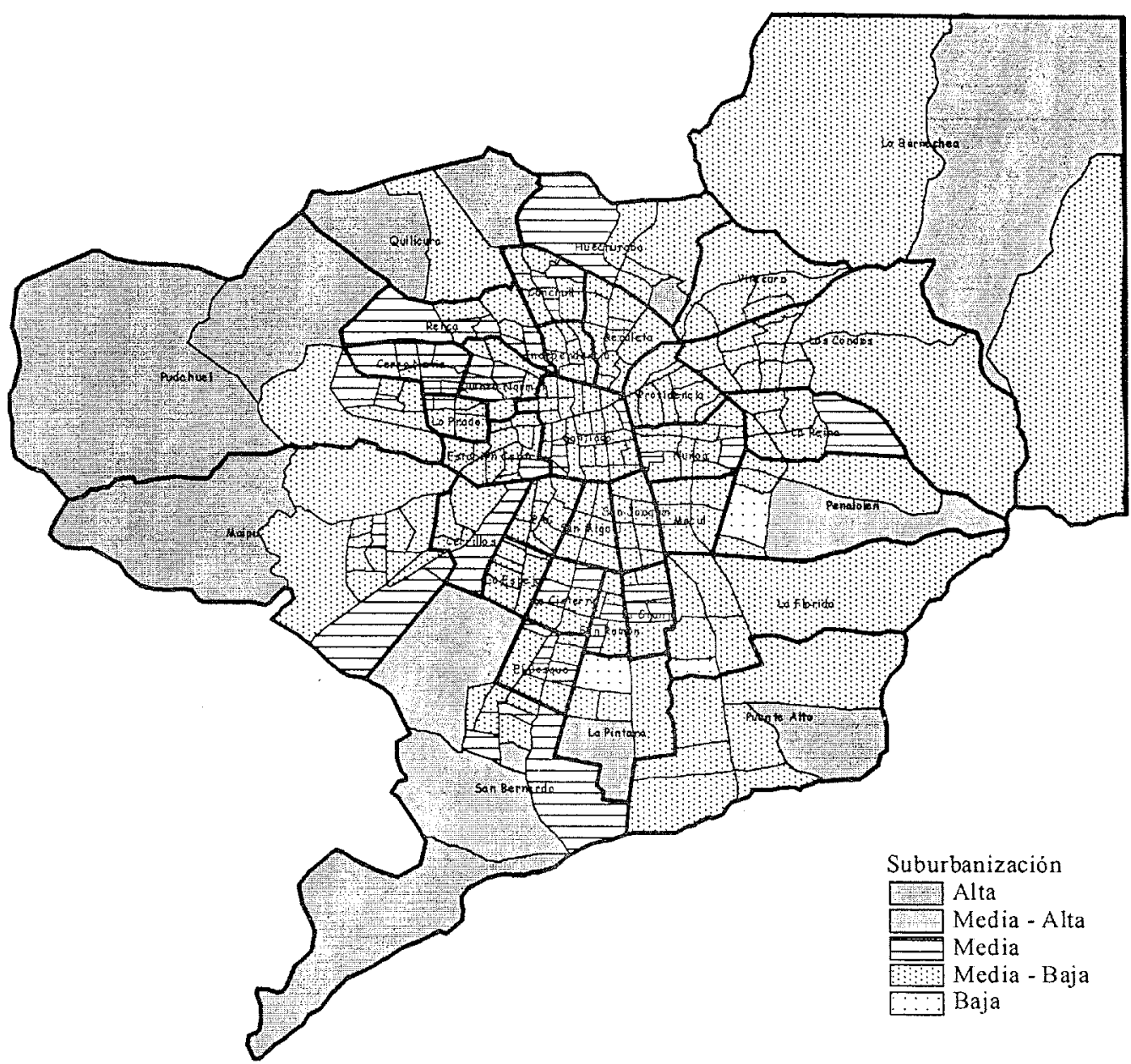

ESCALA APROX. 1:300.000

$\$$

Figura 2.- Gran Santiago. Patrón Suburbanización. 1992.

(Fuente: Inostroza, 1998) 


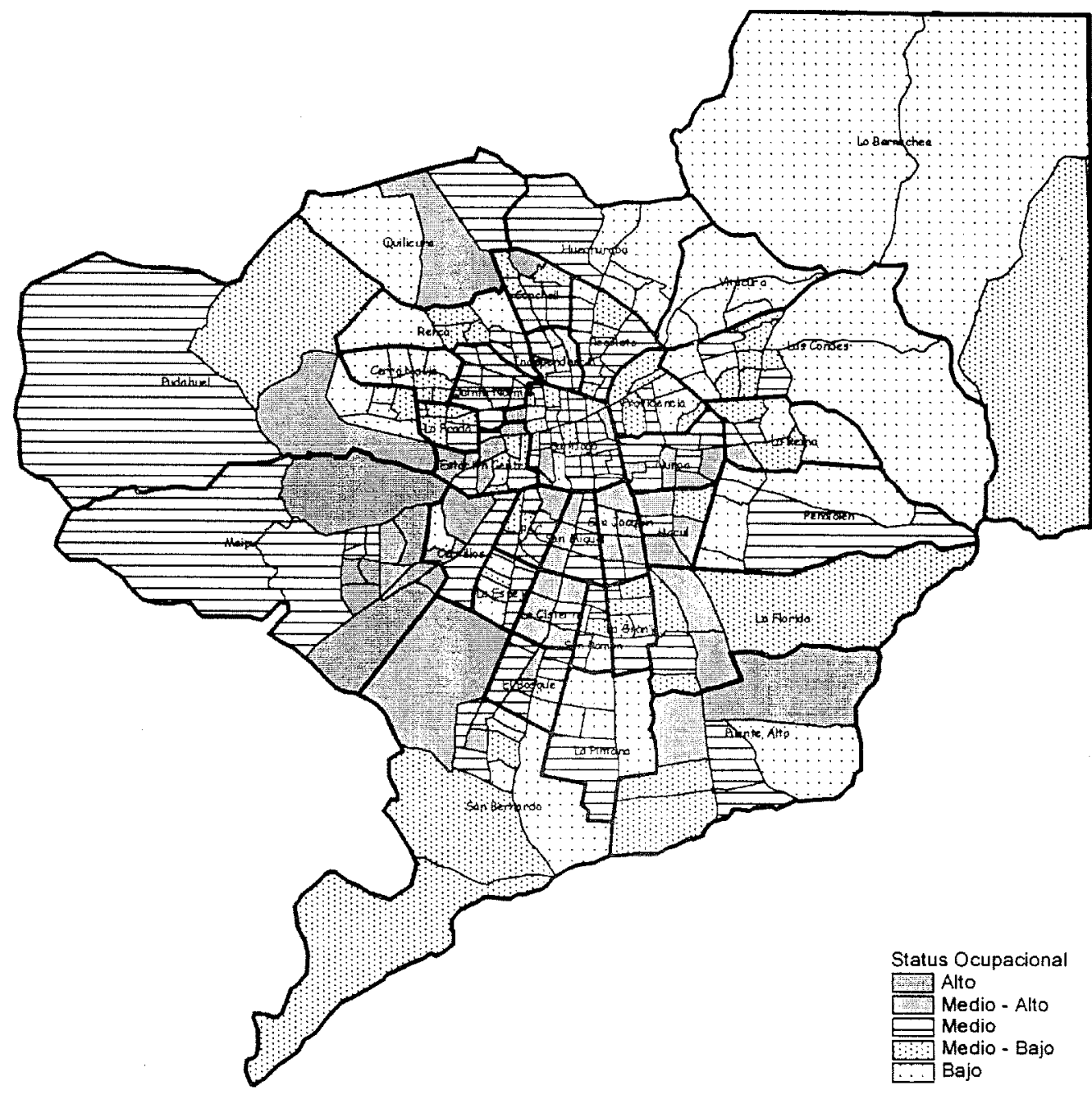

ESCALA APROX. 1: 300.000

3

Figura 3.- Gran Santiago. Patrón Nivel Ocupacional. 1992.

(Fuente: Inostroza, 1998) 
En cambio hacia la periferia, independiente del status socioeconómico de los distritos, la estructura preponderante queda reflejada en un gran anillo concéntrico tipificado por hogares grandes, compuestos por 5 y 6 personas y en donde el tipo dominante de viviendas es la unifamiliar

En general, este patrón es recurrente en numerosos estudios efectuados en ciudades de carácter metropolitano, no sólo desde el punto de vista de su expresión espacial (dicotomía centro-periferia), sino que también en cuanto a las variables que lo conforman. Al respecto, es sabido que en el caso de Santiago, las entidades administrativas que ocupan posiciones más centrales han venido creciendo a tasas negativas en las últimas décadas, proceso que más que detenerse ha terminado por involucrar en el tiempo a más municipios espacialmente colindantes, pese a acciones comunales que intentan revertir esta situación vía renovación urbana y políticas de repoblamiento de espacios centrales.

5.- Patrón Vivienda: La Figura 5 da cuenta de la más alta proporción de distritos centrales con predominio de viviendas cuyo material de las paredes es de adobe, el piso de parquet y la tenecia dominante de ellas, el arriendo.

Correlacionado con la dimensión latente familiar, este factor viene a ratificar lo expresado en la literatura, en el sentido que los espacios más centrales son los sectores de la ciudad de más baja densidad poblacional residente, dominio de las viviendas multifamiliares y arrendadas, alta proporción de población migrante y áreas en franco proceso de deterioro urbano. Para algunos autores, esto se explica en virtud del llamado "engorde de tierra" que llevan adelante algunos propietarios, a la espera que sus predios se valoricen aún más en el tiempo, producto de la expansión del distrito comercial central (C.B.D.) de las ciudades.

Diferenciación del espacio social del Gran Santiago: Mediante la técnica del análisis de agrupamientos, se logró clasificar los 277 distritos del Gran Santiago en 16 clases tal como se observa en la Figura 6.

El patrón espacial resultante muestra claramente la "espina dorsal" correspondiente a los sectores de status socioeconómico más altos, en tanto que el centro de la ciudad se estructura por hogares pequeños y viviendas multifamiliares. Inmediatamente en torno a estos distritos se asocia un gran anillo concéntrico, caracterizado por la presencia de población que desde el punto de vista de su actividad ocupacional, viene a corresponder a una categoria laboral de status medio, al igual que su nivel de instrucción educacional. 


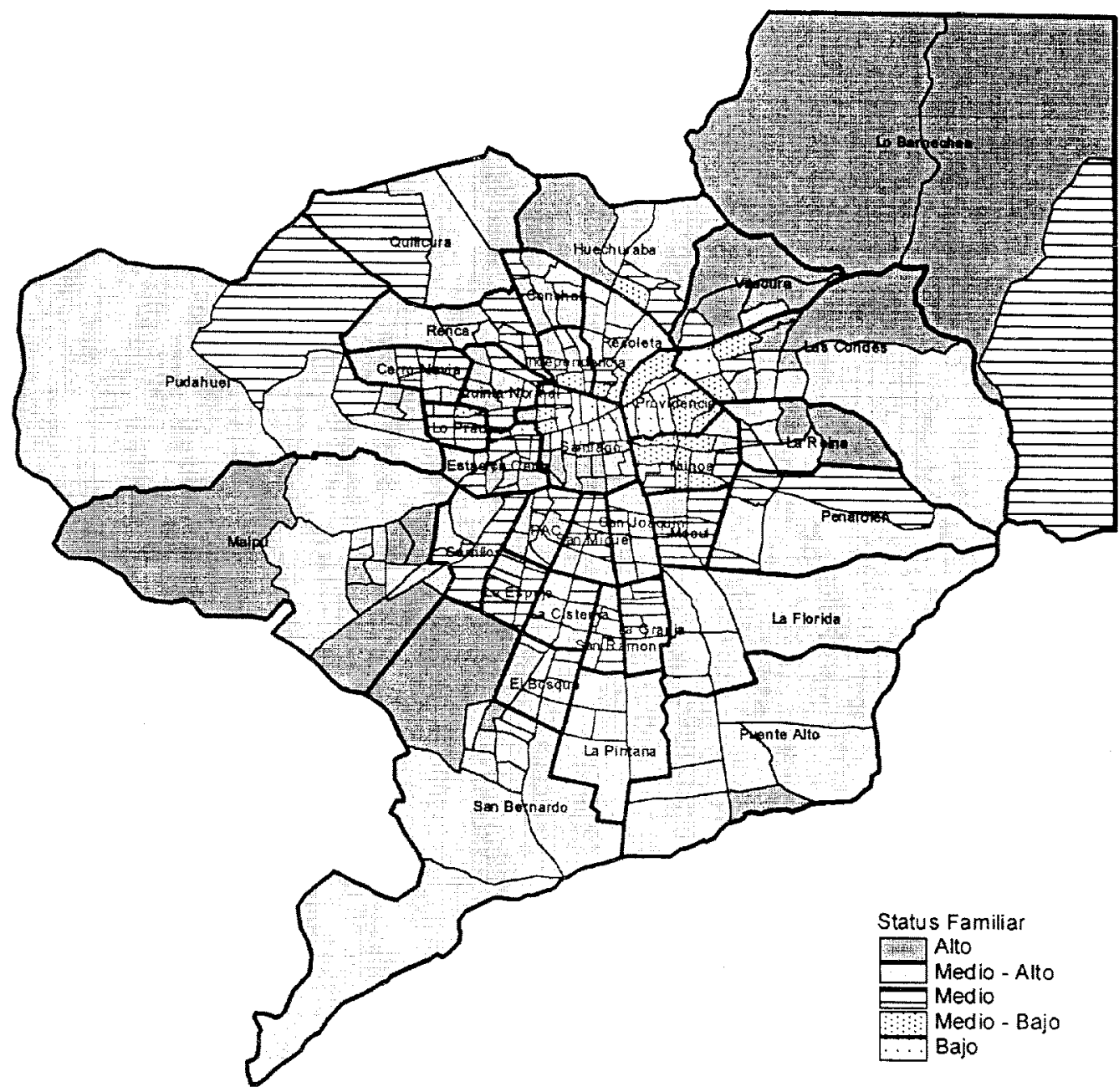

ESCALA: $1: 300.000$

1

Figura 4.- Gran Santiago. Patrón Familiar. 1992.

(Fuente: Inostroza, 1998) 


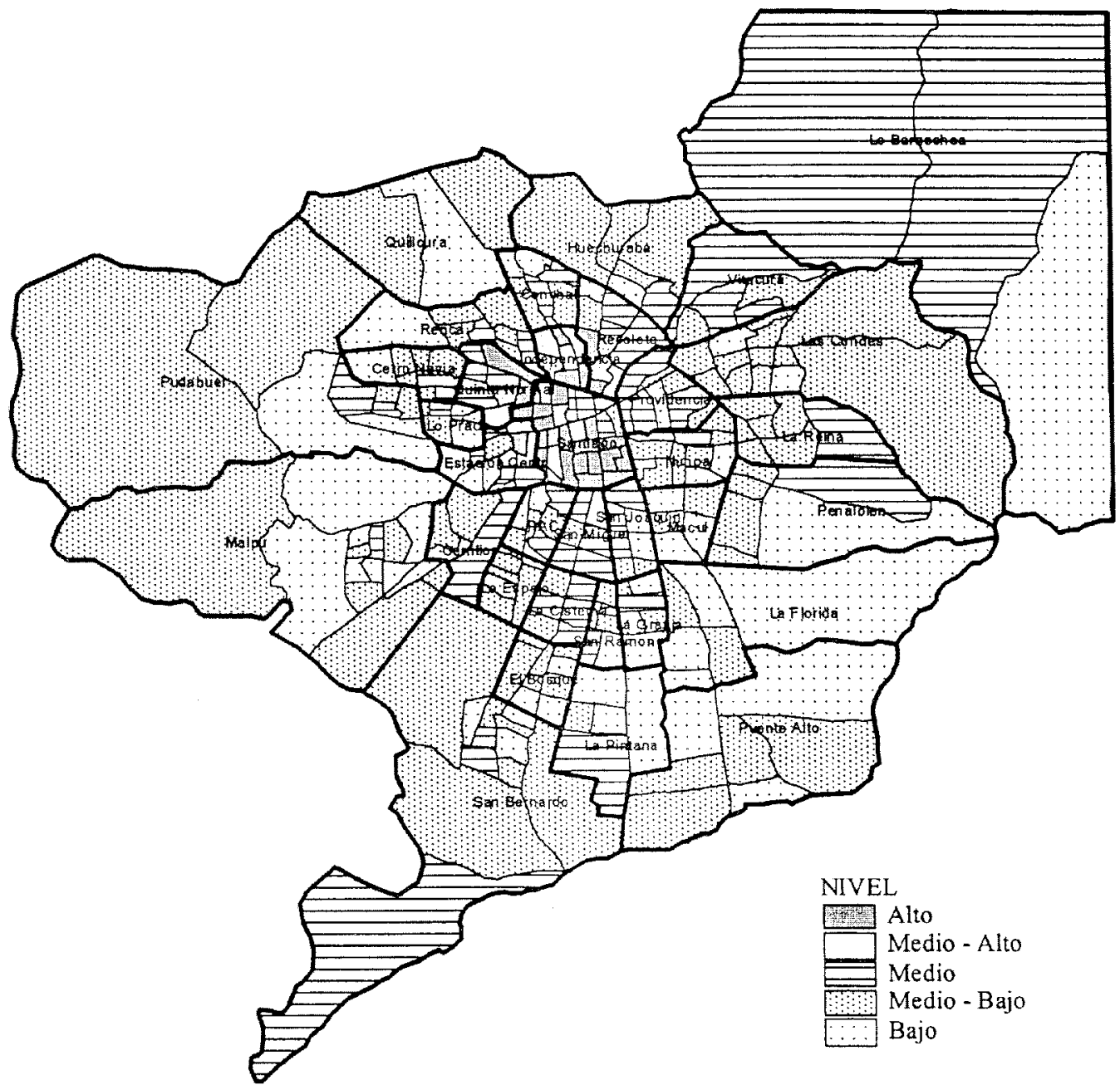

ESCALA APROX. 1:300.000

1

Figura 5.- Gran Santiago. Patrón vivienda. 1992.

(Fuente: Inostroza, 1998) 
1. Empleados de oficina y Foblación con estudios medios

2. Trabajadores no calificados, construcción, servicio doméstico en hogares privados. Dueñas de casa, artesanos y operarios

3. Trabajadores no calificados, construcción, servicio doméstico en hogares privados. Dueñas de casa, artesanos y operarios

4. Departamentos arrendados, hogares pequeños, pocos niños

5. Empleados de oficina, población con estudios medios y técnicos. Viviendas piso piástico, mujeres fértiles.

6. Profesionales, Directivos poderes Ejecutivo y Legislativo, población servicio doméstico, mujeres con ingreso, alto equipamiento.

7. Empleados de oficina, Población con esiudios medios y técnicos. Vivienas tipo casa, hogares numerosos, mujeres fértiles.

8. Profesionales, Directivos poderes Ejecutivo y Legislativo, población servicio doméstico, mujeres con ingreso, alto equipamiento

9. Viviendas piso plástico, mujeres fértiles.

10. Viviendas de adobe, piso entablado. Trabajadores no calificados, construcción, servicio doméstico. Viviendas tipo casa.

11. Empleados de oficina, población estudios medios y técricos. Viviensdas piso plástico, mujeres fértiles.

12. Población trabaja en agricultura, viviendas pozo negro abastecidas por agua de pozo o noria y sin energia eléctrica.

13. Población trabaja en agricultura, viviendas pozo negro abastecidas por agua pozo o noria y sin energia eléctrica.

14. Pablación que trabaja en agricultura, viviendas pozo negro abastecida por agua de pozo o noria y sin energia eléctrica.

15. Dueñas de casa, artesanos y operarios, nivel educativo básico, etnia araucana y religión evangélica. Trabajadores no calificados.

15. Dueñas de casa, artesanos y operarios, nivel educativo básico, etnia araucana y religión evangélica. Viviendas tipo departamento.

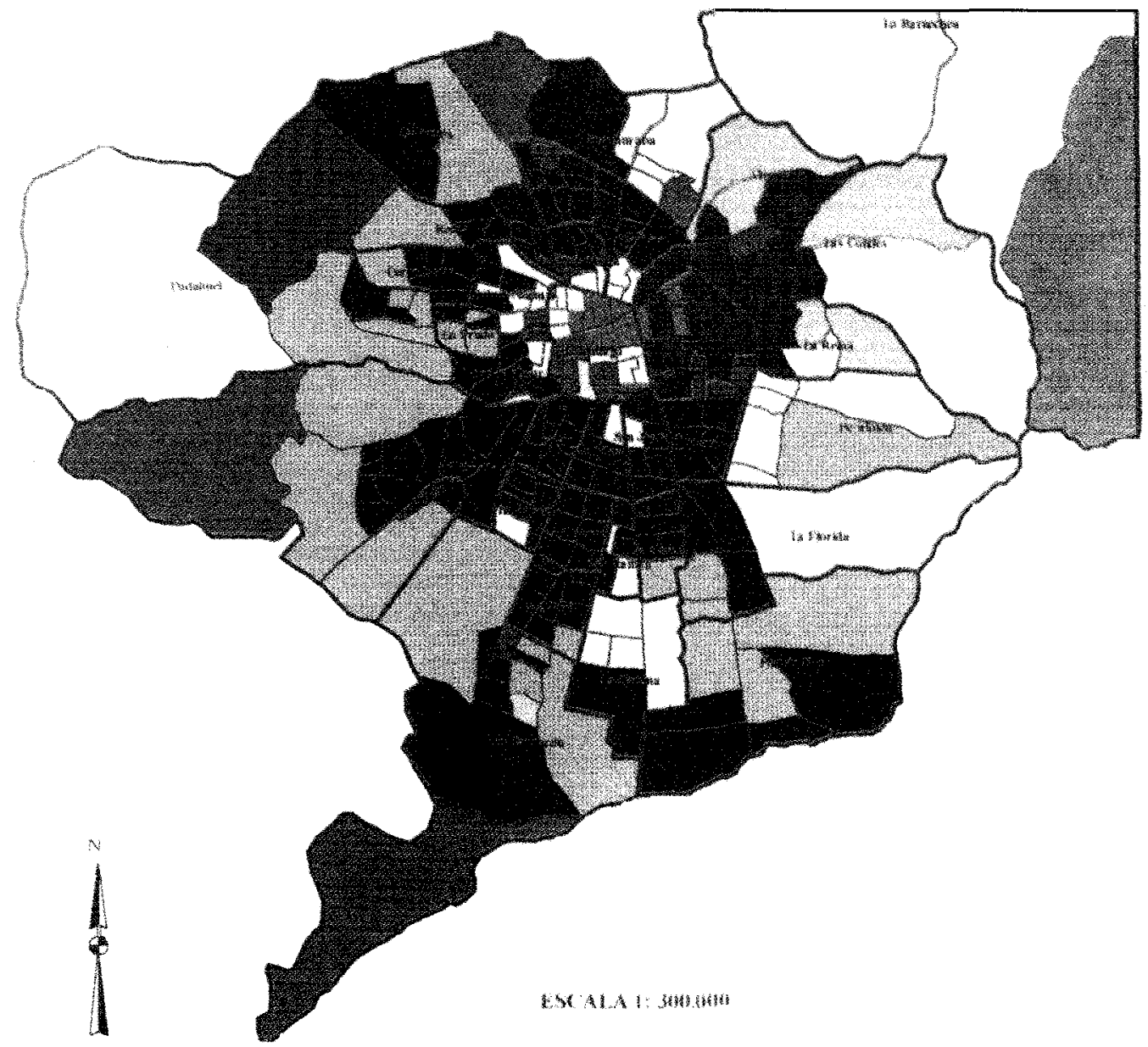

Figura 6.- Diferenciación del espacio social en el Gran Santiago. 1992. (Fuente: Inostroza, 1998) 
Colindante a este anillo, se asocia otro más exterior, tipificado por la presencia de trabajadores no calificados, que laboran en construcción y en donde las mujeres se emplean preferentemente en labores de servicio doméstico en hogares particulares.

Finalmente, el mapa social de la capital nacional, muestra distritos francamente periféricos caracterizados por bajos niveles de equipamiento social básico, patrón que en poco difiere de los modelos sociecológicos planteados para ciudades metropolitanas de latinoamérica.

\section{CONCLUSIÓN}

Del análisis efectuado, queda en evidencia lo "quebrado" del espacio social del Gran Santiago. En efecto, la fuerte segregación socioespacial con una expresión territorial claramente identificable, lleva a hacer planteamientos que en algunos casos han sido títulos de textos y artículos, como por ejemplo, "Santiago dos ciudades", o bien cuando se reconoce al interior de ella "una ciudad rica y otra pobre".

Este patrón de diferenciación del espacio social de una ciudad de carácter metropolitano, se adapta a planteamientos modélicos del espacio urbano de las grandes ciudades, tal como lo han reflejado los planteamientos de GRIFFIN y FORD (1980), BARH y MERTINS (1982) y más recientemente FORD (1996). Es decir, a 1 a concentración sectorial de la población de status socioeconómico más alto, en áreas donde la "spine" (espina dorsal del CBD) se proyecta a través de espacios dominados por la elite residencial de la ciudad. En el caso de Santiago, son evidentes los distritos de la tradicionales comunas de Providencia, Ñuña, Las Condes, La Reina, Vitacura y Lo Barnechea.

En el otro extremo social y espacial, se encuentran los distritos de poblamiento periférico o bien de crecimiento in situ (antiguas áreas excéntricas de la ciudad), que muestran una expresión espacial mezcla sectorial y concéntrica de los atributos que los tipifican. Estas entidades territoriales, constituyen la mayor proporción en el conjunto del sistema de distritos del Gran Santiago, ratificando con ello la fuerte segregación social urbana que se dan en las grandes metrópolis, particularmente de los países en vía de desarrollo, no siendo la capital de Chile una excepción a esta realidad.

\section{BIBLIOGRAFÍA}

BÄRH, J. y RIESCO, R. (1981): "Estructura urbana de las metrópolis latinoamericanas. El caso de la ciudad de Santiago". Revista de Geografia Norte Grande, 8, 27-55. 
BÄRH, J y MERTINS, G. (1982): “A model of the social and spatial differentiation of latin american metropolitan cities". Applied Geography and Development, 19, 22-45.

BÁRH, J. y MERTINS, G. (1993): "La ciudad en América Latina". Revista Población y Sociedad, 1, 5-14.

BERRY, B.J.L. (comp.) (1975): “Comparative factorial ecology”. Economic Geography 47, 2.

BOADA, C. (1985): "Algunas consideraciones sobre los estudios ecológicos en áreas urbanas". Revista Geográfica Venezolana. Universidad de Los Andes, Mérida, Venezuela, 9, 5-31.

FORD L. (1996): “A new and improved model of latin american city structure”. The Geographical Review, 86 (3), 437-440.

GRIFFIN, E y FORD L. (1980): "A model of latin america city structure". Geographical Review, 70, $397-422$.

INOSTROZA, W. (1998): Distribución espacial del status ocupacional de la población en el Gran Santiago: Un análisis de la diferenciación del espacio social. Memoria para optar al título de Geógrafo. Universidad de Chile. 91 p.

ORTIZ, J. (1980): Evolución de la diferenciación areal interna del espacio social de El Vigía, 19651979. Memoria para optar al grado de Magister Scientiae. Universidad de Los Andes, Mérida, Venezuela, $149 \mathrm{p}$.

ORTIZ J. y SCHIAPPACASSE, P. (1997): "Las migraciones intraurbanas y su impacto en la diferenciación del espacio social del Gran Santiago". Revista Geográfica Terra Australis, 42, 121-138.

SCHIAPPACASSE, P. (1998): Diferenciación del espacio social intraurbano en el Gran Santiago. Un análisis a nivel distrital. Tesis para optar al grado de Magister en Geografía, Universidad de Chile. 151 p.

THEODORSON, G.A. (com.) (1974): Estudios de ecología humana. Biblioteca Universitaria Labor, Barcelona. 2 vol.

TIMMS, D. (1976): "El mosaico urbano. Hacia una teoría de la diferenciación residencial". Instituto de Estudios de Administración local, Colección Nuevo Urbanismo. 21, 458 p. 